\title{
Analytical solution of the concentration and current in the electoenzymatic processes involved in a PPO-rotating-disk-bioelectrode
}

\author{
Govindhan Varadharajan, Lakshmanan Rajendran*
}

Department of Mathematics, The Madura college, Madurai, India; ${ }^{*}$ Corresponding Author: raj_sms@rediffmail.com

Received 16 October 2010; revised 20 November 2010; accepted 25 November 2010.

\begin{abstract}
A mathematical model for electroenzymatic process of a rotating-disk-bioelectrode in which polyphenol oxidase occurs for all values of concentration of catechol substrate is presented. The model is based on system of reaction-diffusion equations containing a non-linear term related to Michaelis-Menten kinetics of the enzymatic reaction. Approximate analytical method (He's Homotopy perturbation method) is used to solve the non-linear differential equations that describe the diffusion coupled with a Michaelis-Menten kinetics law. Closed analytical expressions for substrate concentration, product concentration and corresponding current response have been derived for all values of parameter $\gamma_{E}\left(=\gamma L^{2}\right)$ and $\alpha_{1}\left(=\alpha S_{\infty}\right)$ using perturbation method. These results are compared with simulation results and are found to be in good agreement. The obtained results are valid for the whole solution domain.
\end{abstract}

Keywords: Mathematical Model; Polyphenol Oxidase; Steady-State; Homotopy Perturbation Method; Simulation

\section{INTRODUCTION}

Enzymes electrodes are powerful tools for understanding the mechanism and kinetics of fast reactions. Owing to their specificity and sensitivity, enzyme electrodes including various amplification, schemes have been developed for many applications such as electrochemical immunoassays, [1-2] water pollutant detection, [3-7] and monitoring of biological metabolities [8-11]. The sensitivity of enzyme electrodes is very often increased by incorporation of a substrate-recycling scheme and several strategies including chemical, enzymatic, or electrochemical recycling have been developed. In the view of numerous application of such bio-sensor with amplified response, we are interested in investigating the concentration $\mathrm{s}$ and $\mathrm{p}$ in order to improve the metrological characteristics further. In addition, this theoretical approach is of practical interest since this kind of biosensor can be used for the determination of phenolic compounds and catecholamine neurotransmitters in the field of environmental control and clinical analysis [1222]. Such a theoretical and kinetic analysis is a powerful approach to rationalize functions of biosensors. Desprez and Labbe [23] obtained the analytical expression concentration and current for the limiting cases only. The purpose of this communication is to derive a simple accurate polynomial expressions of concentrations generated at a enzyme electrode using Homotopy perturbation method.

\section{MATHEMATICAL FORMULATION OF THE PROBLEM}

Figure 1 is a schematic representation of the rotating disk PPO-electrode working with catechol S substrate. The different assumption which lead to the electrode

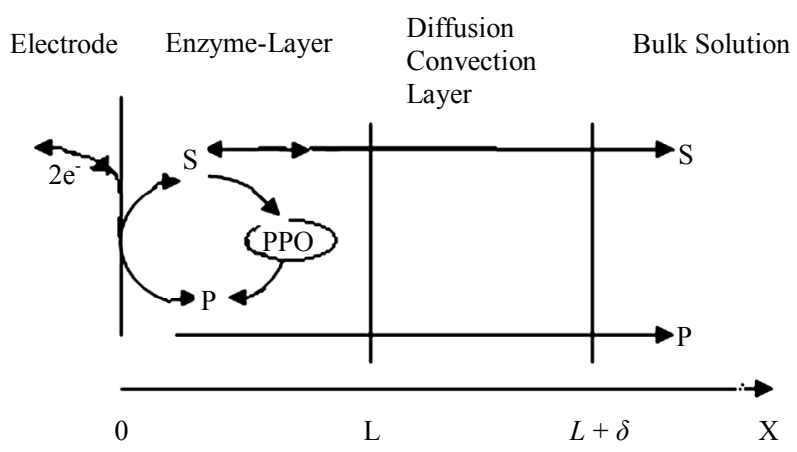

Figure 1. Schematic representation for the electrocatalytic process that occur at PPO-rotating-disk-bio electrode. $\mathrm{s}$ and $\mathrm{p}$ respectively denote catechol substrate and product of PPO. L and $\delta$ are respectively the thickness of the enzyme-layer and the diffusion-convection layer. 
response are as given below: 1) In our model we have assumed that, because of their structural similarity, s and $\mathrm{p}$ have the same diffusion coefficient D' in the bulk solution. 2) Diffusion of $s$ and $p$ within the enzymes layer of thickness $\mathrm{L}$ with the same diffusion coefficient $\mathrm{D}$ and a partition coefficient equal to unity. 3) Enzymatic reaction between substrate $\mathrm{s}$ and enzymes $\mathrm{E}$ within the enzyme layer. The rate of the enzymatic step is $\gamma=k_{\text {cat }}[E]_{T}[S] / K_{S}+[S]$, Where $K_{S}$ is the Michaelis constant for substrate $\mathrm{s}$ and $k_{\text {cat }}$ the rate of the enzymatic rate-limiting step in the Michaelis-Menten formalism. $[E]_{T}$ is the total concentration of enzyme within the enzyme layer.(iv) Depending on the applied electrode potential, electroreduction of enzymatically produced orthoquinone $\mathrm{p}$ or electro-oxidation of non-enzymatically oxidized substrates occurs on the electrode surface. Electrode potential values are assumed to be sufficiently Cathodic or anodic so that this electrochemical is not rate limiting. We consider the equations of Barlett and Whitaker [24], Desprez and Labbe [23], describing the concentrations of $s$ and $p$ at steady state as follows:

$$
\begin{aligned}
& \frac{d^{2} S}{d x^{2}}-\frac{\gamma S}{1+\alpha S}=0 \\
& \frac{d^{2} P}{d x^{2}}+\frac{\gamma S}{1+\alpha S}=0
\end{aligned}
$$

where

$$
\gamma=\frac{1}{\Lambda^{2}}, \alpha=\frac{1}{K_{S}}, \Lambda=\left[\frac{D K_{S}}{k_{c a t}(E)_{T}}\right]^{\frac{1}{2}}
$$

Using the following boundary conditions:

$$
\begin{aligned}
& S=S_{\infty}, P=P_{\infty}=0 \quad \text { when } \quad x \geq L+\delta \\
& S=0, P=0 \quad \text { when } \quad x=0,
\end{aligned}
$$

We introduce the following set of dimensionless variables:

$$
U=\frac{S}{S_{\infty}}, V=\frac{P}{P_{\infty}}, X=\frac{x}{L}, \gamma_{E}=\gamma L^{2}, \alpha_{1}=\alpha S_{\infty}
$$

The governing non-linear reaction/diffusion Eqs.1-5 is expressed in the following non- dimensional form as :

$$
\begin{aligned}
& \frac{d^{2} U}{d X^{2}}-\frac{\gamma_{E} U}{1+\alpha_{1} U}=0 \\
& \frac{d^{2} V}{d X^{2}}+\frac{\gamma_{E} U}{1+\alpha_{1} U}=0
\end{aligned}
$$

with the boundary conditions:

$$
\begin{gathered}
V=0, U=1, \text { when } X=1+\frac{\delta}{L} \\
V=0, U=1, \text { when } X=0
\end{gathered}
$$

The dimensionless current is given by

$$
\psi=I_{a} / 2 F A D S_{\infty}=(d U / d X)_{X=0}
$$

\section{ANALYTICAL SOLUTION OF STEADY STATE CONCENTRATION USING HPM}

Recently, many authors have applied the HPM to various problems and demonstrated the efficiency of the HPM for handling non-linear structures and solving various physics and engineering problems [25-28]. This method is a combination in topology and classic perturbation techniques. Ji Huan He used the HPM to solve the Lighthill equation [29], the Duffing equation [30] and the Blasius equation [31]. The idea has been used to solve non-linear boundary value problems, integral equations and many other problems [32-33]. The HPM is unique in its applicability, accuracy and efficiency. The HPM uses the imbedding parameter $p$ as a small parameter and only a few iterations are needed to search for an asymptotic solution. Using this method (see Appendix A), we can obtain the following solution to Eqs.7 and 8 (Appendix-A)

$$
\begin{aligned}
& U(X) \\
& =1+\frac{1}{24}\left[\left(\gamma_{E}^{2}\left(1+\frac{\delta}{L}\right)^{3}+12 \alpha_{1} \gamma_{E}\left(1+\frac{\delta}{L}\right)-12 \gamma_{E}\left(1+\frac{\delta}{L}\right)\right) X\right. \\
& \left.+12 \gamma_{E}\left(1-\alpha_{1}\right) X^{2}-2 \gamma_{E}^{2}\left(1+\frac{\delta}{L}\right) X^{3}+\gamma_{E}^{2} X^{4}\right] \\
& V(X) \\
& =\frac{1}{24}\left[\left(12 \gamma_{E}\left(1+\frac{\delta}{L}\right)-12 \gamma_{E} \alpha_{1}\left(1+\frac{\delta}{L}\right)-\gamma_{E}^{2}\left(1+\frac{\delta}{L}\right)^{3}\right) X\right. \\
& \left.+12 \gamma_{E}\left(\alpha_{1}-1\right) X^{2}-\gamma_{E}^{2} X^{4}+2 \gamma_{E}^{2}\left(1+\frac{\delta}{L}\right) X^{3}\right]
\end{aligned}
$$

The current response is given by

$$
\begin{aligned}
\psi= & I_{a} / 2 F A D S_{\infty} \\
= & 0.5 \gamma_{E}(1+\delta / L)-0.5 \gamma_{E} \alpha_{1}(1+\delta / L) \\
& -\gamma_{E}^{2}(1+\delta / L)^{3}
\end{aligned}
$$

\section{LIMITING CASE RESULTS}

The kinetic response of amperometric biosensor de- 
pends on concentrations of $U$ and $V$. The concentrations of the species depend upon concentration of the substrate $U$. But substrate $U$ depends on two factors $\gamma_{E}$ and $\alpha_{1}$. $K_{S}$ is the Michaelis-Menten constant, an intrinsic character of an enzyme.

\subsection{Unsaturated (First Order) Catalytic Kinetics}

When $\alpha_{1} U \gg 1$, the reaction rate can be simplified to $v=k_{\text {cat }}[E]_{T}$ as a zero reaction. In zero order reaction $\alpha_{1} U$ is small. Now the Eq.7 becomes

$$
\frac{d^{2} U}{d X^{2}}-\frac{\gamma_{E}}{\alpha_{1}}=0
$$

The solution of the above equation becomes

$$
U=1+\frac{\gamma_{E} X^{2}}{2 \alpha_{1}}-\frac{\gamma_{E}}{2 \alpha_{1}}\left(1+\frac{\delta}{\mathrm{L}}\right) X
$$

\subsection{Unsaturated (Zero Order) Catalytic Kinetics}

If $\alpha_{1} U \ll 1$, the rate will be $v=k_{\text {cat }}[E]_{T}[S] / K_{S}$ as a first order reaction. Now the Eq.7 becomes

$$
\frac{d^{2} U}{d X^{2}}-\gamma_{E} U=0
$$

From the above equation we can obtain the concentration of $U$ as follows:

$$
\begin{aligned}
U & =\left(\frac{2 \cosh \sqrt{\gamma_{\mathrm{E}}}\left(1+\frac{\delta}{L}\right)-\mathrm{e}^{\sqrt{\gamma_{\mathrm{E}}}\left(1+\frac{\delta}{L}\right)}+1}{2 \cosh \sqrt{\gamma_{\mathrm{E}}}\left(1+\frac{\delta}{L}\right)}\right) \mathrm{e}^{\sqrt{\gamma_{E}} X} \\
& +\left(\frac{\mathrm{e}^{\sqrt{\gamma_{\mathrm{E}}}\left(1+\frac{\delta}{\mathrm{L}}\right)}-1}{2 \cosh \sqrt{\gamma_{\mathrm{E}}}\left(1+\frac{\delta}{L}\right)}\right) \mathrm{e}^{-\sqrt{\gamma_{\mathrm{E}}} X}
\end{aligned}
$$

\section{NUMERICAL SIMULATION}

The non-linear differential Eq.7 is also solved using numerical methods. The functionbvp4c in Scilab software which is a function of solving two-point boundary value problems (BVPs) for ordinary differential equations is used to solve this equation. It's numerical solution is compared with Homotopy perturbation method and it gives satisfactory result. The Scilab program is also given in Appendix (B).

\section{RESULTS AND DISCUSSION}

In other cases the order is between zero and one. For an enzyme electrode to be analytically useful, its response must be quantitatively related to the substrate concentration. Based on this principle, $\alpha_{1} U \gg 1$ is not the proper case for an enzyme electrode, because in the zero order reaction product concentration is independent of the substrate concentration. An order between zero and one is favorable. Eq. 12 represents the most general approximate new analytical expression for the substrate concentration profiles for all values of $\gamma_{E}$ and $\alpha_{1}$. Recently Labbe et al. [23] obtained the solution of this model for the limiting cases. A comparison of numerical simulation results with our Eq.12 is shown in Figures 2(a-c). The agreement between simulation results and Eq.12 is quite good. Figure 3(a-c) show the dimensionless steady-state concentration $V$ using Eq.13 for various values of $\gamma_{E}$ and $\alpha_{1}$. From these figures, we can see that the value of the concentration $V$ matches well with the simulation results. The concentration $U$ attains minimum or $V$ attains maximum when

$$
X=\frac{12\left(1+\frac{\delta}{L}\right)-\gamma_{E}\left(1+\frac{\delta}{L}\right)^{3}-12 \alpha_{1}\left(1+\frac{\delta}{L}\right)}{24\left(1-\alpha_{1}\right)}
$$

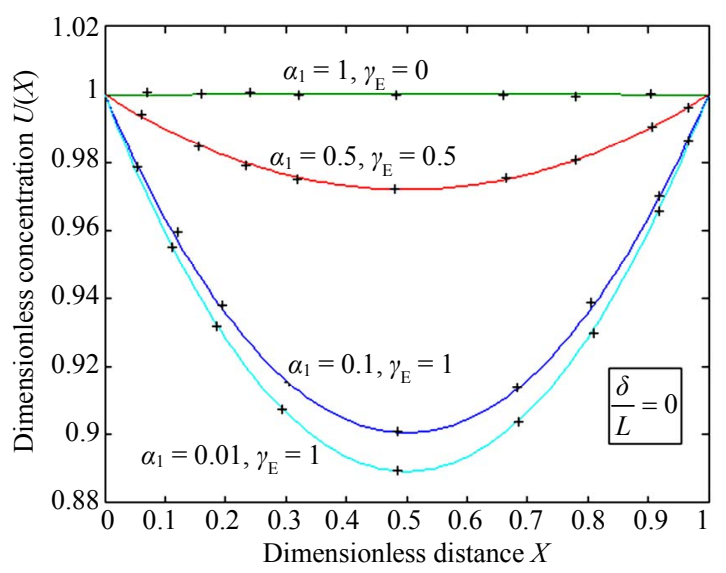

(a)

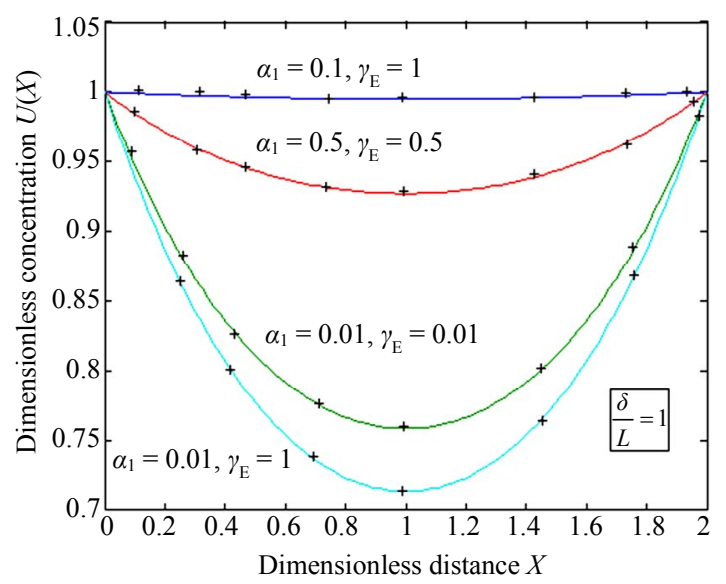

(b) 


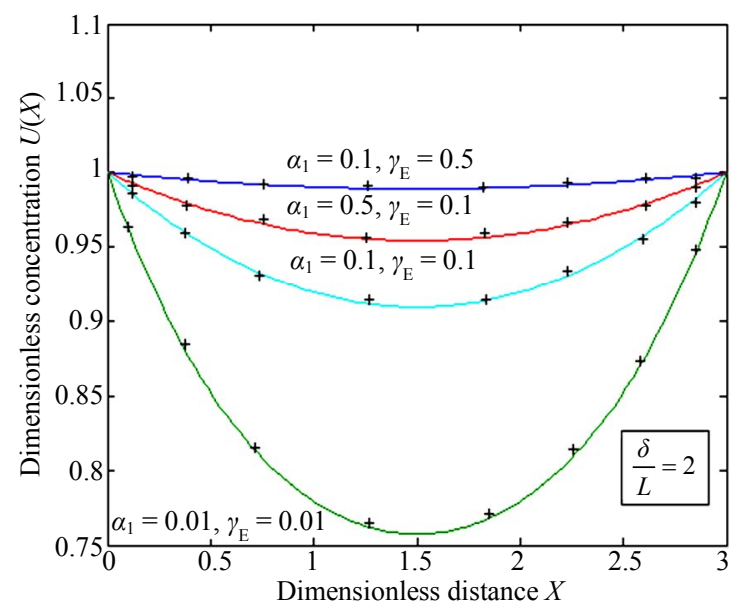

(c)

Figure 2. Normalised concentration profile $U(X)$ as a function of dimensionless parameter $X=x / L$. The concentrations were computed using Eq.12 for various values of the reaction/ diffusion parameter $\alpha_{1}, \gamma_{\mathrm{E}}$ and for the values (a) $\delta / L=0$ (b) $\delta / L=1$ (c) $\delta / L=2$. The line denotes Eq.12 and the dot denotes the numerical simulation.

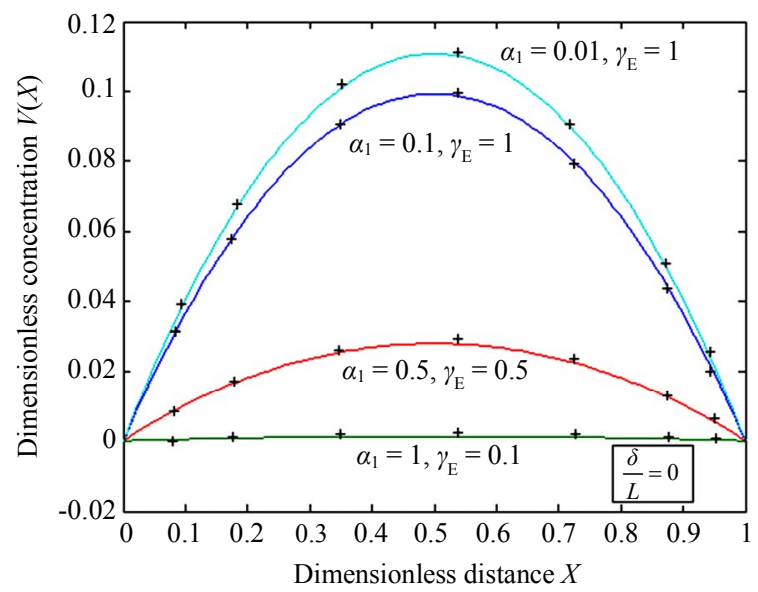

(a)

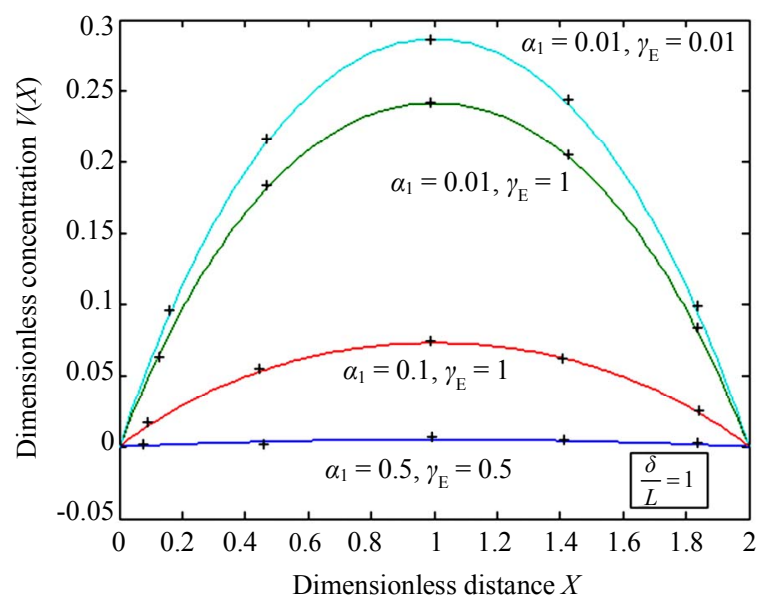

(b)

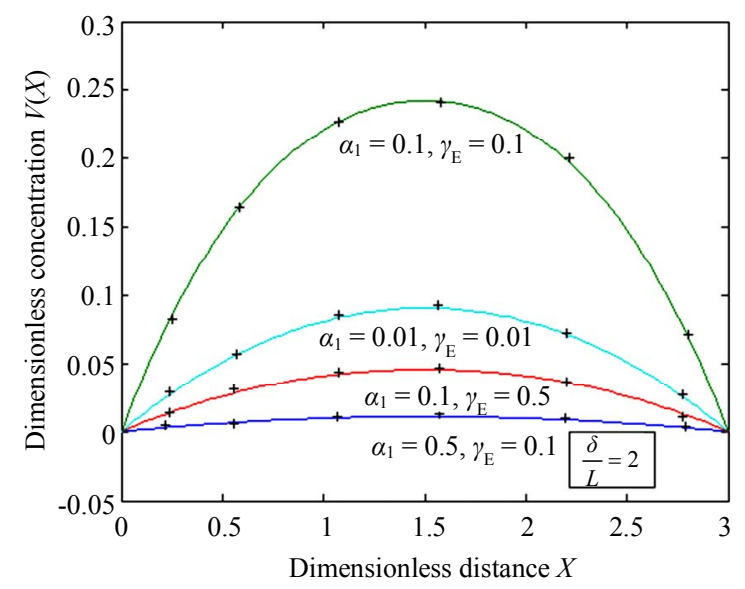

(c)

Figure 3. Normalised concentration profile $V(X)$ as a function of dimensionless parameter $X=x / L$. The concentrations were computed using Eq.13 for various values of the reaction/ diffusion parameter $\alpha_{1}, \gamma_{\mathrm{E}}$ and for the values (a) $\delta / L=0$ (b) $\delta / L=1$ (c) $\delta / L=2$ The line denotes Eq.12 and the dot denotes the numerical simulation.

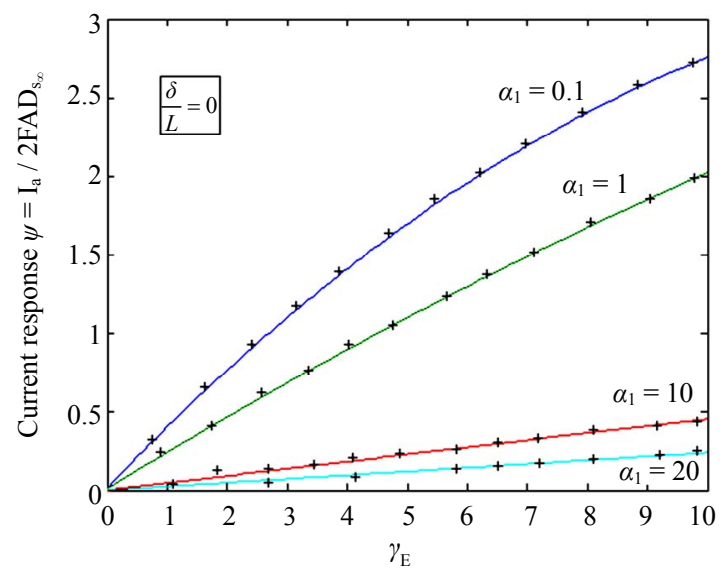

Figure 4. Dimensional current $\psi$ versus $\gamma_{\mathrm{E}}$ for various values of $\alpha_{1}$ and for the fixed value $\delta / L=0$ The line denotes Eq.14 and the dot denotes the numerical simulation.

Figure 4 represents the dimensionless current $\Psi$ versus $\gamma_{E}$ for various values of $\alpha_{1}$. From this figure, it is observed that the value of the current increases slowly when $\gamma_{E}$ increases and $\alpha_{1}$ decreases. Our analytical expression of current is also compared with simulation result in Figure 4. It gives satisfactory agreement.

\section{CONCLUSIONS}

The time independent non-linear reaction-diffusion equation has been formulated and solved analytically and numerically. Analytical expressions for the concentrations and current are derived by using the HPM. The 
primary result of this work is simple approximate calculations of concentrations and current for all values of dimensionless parameters $\alpha_{1}, \gamma_{E}$ and $\delta / L$. The HPM is an extremely simple method and it is also a promising method to solve other non-linear equations. This method can be easily extended to find the solution of all other non-linear equations.

\section{ACKNOWLEDGEMENTS}

The authors are very grateful to the referees for their valuable suggestions. This work was supported by the Department of Science and Technology (DST), New Delhi, Government of India. The authors are also thankful Dr. T. V. Krishnamoorthy, The Principal and Mr. M.S Meenakshisundaram, The Secretary, The Madura College, Madurai for their encouragement.

\section{REFERENCES}

[1] Kronkvist, K., Wallentin, K., Johansson, G. (1994) Selective enzyme amplification of $\mathrm{NAD}^{+} / \mathrm{NADH}$ using coimmobilized glycerol dehydrogenase and diaphorase with amperometric detection. Analytical chimica Acta, 290-335.

doi:10.1016/0003-2670(94)80120-7

[2] Kotte, H., Grundig, B., Vorlop, K. D., Strehlitz, B. and Stottmeister, U. (1995) Methylphenazonium-modified enzyme sensor based on polymer thick films for subnanomolar detection of phenols. Analytical chemistry, 67, 65-70.

doi:10.1021/ac00097a011

[3] Wang, J., Lu, J., Ly, S.Y., Vuki, M., Tian, B., Adeniyi, W.K. and Armendariz, R.A. (2000) Lab-on-a-Cable for electrochemical monitoring of phenolic contaminants. Analytical chemistry, 72, 2659-2663. doi:10.1021/ac991054y

[4] Zhenjiu, L., Deng, J. and Li. D. (2000) A new tyrosinase biosensor based on tailoring the porosity of $\mathrm{Al}_{2} \mathrm{O}_{3}$ sol-gel to co- immobilize tyrosinase and the mediator. Analytical chimica Acta, 407, 87-96. doi:10.1016/S0003-2670(99)00807-7

[5] Russell, I. M. and Burton, S. G. (1999) Development and demonstration of an immobilised-polyphenol oxidase bioprobe for the detection of phenolic pollutants in water. Analytical chimica Acta, 389, 161-170. doi:10.1016/S0003-2670(99)00143-9

[6] Cosnier. S., Fombon. J.J., Labbe, P. and Limosin, D. (1999) Development of a PPO-poly (amphiphilic pyrrole) electrode for on site monitoring of phenol in aqueous effluents. Sensors and Actuators B, 59, 134-139.

[7] Nistor, C., Emneus, J., Gorton, L. and Ciucu, A. (1999) Improved stability and altered selectivity of tyrosinase based graphite electrodes for detection of phenolic compounds. Analytical chimica Acta, 387, 309-326. doi:10.1016/S0003-2670(99)00071-9

[8] Forzani, E.S., Rivas, G.A. and Solis, V.M. (1999) Kinetic behaviour of dopamine-polyphenol oxidase on electrodes of tetrathiafulvalenium tetracyanoquinodimethanide and tetracyanoquinodimethane species V. M. Journal of Electroanalytical Chemistry, 461, 174-183.
doi:10.1016/S0022-0728(98)00119-3

[9] Moore, T.J., Nam, G.G., Pipes, L.C. and Coury Jr, L.A. (1994) Chemically amplified voltammetric enzyme electrodes for oxidizable pharmaceuticals. Analytical Chemistry, 66, 3158-3163. doi: $10.1021 / \mathrm{ac} 00091 \mathrm{a} 026$

[10] Lisdat, F., Wollenberg, U., Paeschke, M. and Scheller, F.W. (1998) Sensitive catecholamine measurement using a monoenzymatic recycling system. Analytical chimica Acta, 368, 233-241. doi:10.1016/S0003-2670(98)00221-9

[11] Forzani, E.S., Solis, V. and Calvo, E.J. (2000) Electrochemical behavior of polyphenol oxidase immobilized in self-assembled structures layer by layer with cationic polyallylamine. Analytical Chemisty, 72, 5300-5307.

[12] Toyota, T., Kuan, S.S. and Guilbault, G.G. (1985) Determination of total protein in serum using a tyrosinase enzyme electrode, Anaytical chemistry, 57, 1925-1928. doi: $10.1021 / \mathrm{ac} 00286 \mathrm{a} 030$

[13] Kulys, J. and Schmid, R.D. (1990) A Sensitive Enzyme Electrode for Phenol Monitoring. Analytical Letters, 23, 589-597.

[14] Wang, J. and Varughese, K. (1990) Polishable and robust biological electrode surfaces. Analytical chemistry, 62 318-320. doi:10.1021/ac00202a019

[15] Skladal, P. (1991) Mushroom tyrosinase-modified carbon paste electrode as an amperometric biosensor for phenols. Collection of Czechoslovak Chemical Communications, 569, 1427-1433.

[16] Hall, G.F., Best, D.J. and Turner, A.P.F. (1988) The determination of $p$-cresol in chloroform with an enzyme electrode used in the organic phase. Analytical chimica Acta, 213, 113-119. doi:10.1016/S0003-2670(00)81345-8

[17] Cosiner, S. and Innocent, C. (1992) A novel biosensor elaboration by electropolymerization of an adsorbed amphiphilic pyrrole-tyrosinase enzyme layer. Journal of Electroanalytical Chemistry, 328, 361-366. http://dx.doi.org/10.1016/0022-0728(92)80195-A

[18] Wang, J., Nasser, N., Kwon and Cho, M.Y. (1992) Tissue bioelectrode for organic-phase enzymatic assays, Analytical chimica Acta, 2649, 7-12. doi:10.1016/0003-2670(92)85290-M

[19] Ortega, F., Dominguez, E., Pettersson J. and Gorton, L. (1993) Amperometric biosensor for the determination of phenolic compounds using a tyrosinase graphite electrode in a flow injection system. Journal of Biotechnology, 31, 289-300 doi:10.1016/0168-1656(93)90075-X

[20] Besombes, J. L., Cosnier, S., Labbe, P and Reverdy, G. (1995) Determination of Phenol and Chlorinated Phenolic Compounds Based on a PPO-Bioelectrode and Its Inhibition, Analytical Letters, 28, 405-424.

[21] Lutz, M., Burestedt, E., Emneus, J., Liden, H., Gobhadi, S., Gorton, L. and Marko-Varga, G. (1995) Effects of different additives on a tyrosinase based carbon paste electrode, Analytical chimica Acta, 305, 8-17. doi:10.1016/0003-2670(94)00573-5

[22] Onnerfjord, P., Emneus, J., Marko-Varga, G., Gorton, L., Ortega, F. and Dominguez, E. (1995) Tyrosinase graphite-epoxy based composite electrodes for detection of 
phenols, Biosensors Bioelectronics, 10, 607-619. doi:10.1016/0956-5663(95)96937-T

[23] Desprez, V. and Labbe, P. (1996) A Kinetic model for the electroenzymatic processd involved in polyphenol-oxidase- based amperometric catechol sensors. Journal of Electroanalytical Chemistry, 415, 191-195. doi:10.1016/S0022-0728(96)01011-X

[24] Bartlett, P.N. and Whitaker, R.G. (1987) Electrochemical immobilisation of enzymes: Part I. Theory, Journal of Electroanalytical Chemistry, 224, 27-35. doi:10.1016/0022-0728(87)85081-7

[25] Li, S.J. and Liu, Y.X. (2006) An improved approach to nonlinear dynamical system identification using PID neural networks," International Journal of NonlinearScience and Numerical Simulation, 7, 177-182.

[26] Mousa, M.M., Ragab, S.F. and Nturforsch, Z. (2008) Application of the homotopy perturbation method to linear and nonlinear schrödinger equations, Zeitschrift für Naturforschung, 63, 140-144.

[27] He, J.H, Homotopy perturbation technique, Computer Methods in Applied Mechanics and Engineering, vol. 178 (1999) 257-262. doi:10.1016/S0045-7825(99)00018-3

[28] He, J.H. (2003) Homotopy perturbation method: a new nonlinear analytical Technique. Applied Mathematics and Computation, 135, 73-79.

doi:10.1016/S0096-3003(01)00312-5

[29] He, J.H. (2003) A Simple perturbation approach to Blasius equation", Applied Mathematics and Computation, 140, 217-222. doi:10.1016/S0096-3003(02)00189-3

[30] He, J.H. (2006) Some asymptotic methods for strongly nonlinear equations. International Journal of Modern Physics B, 20, 1141-1199. doi:10.1142/S0217979206033796

[31] He, J.H., Wu, G.C. and Austin, F. (2010) The variational iteration method which should be followed. Nonlinear Science Letters A, 1, 1-30.

[32] He, J.H. (2000) A coupling method of a homotopy technique and a perturbation technique for non-linear problems. Internationl Journal of Nonlinear Mechanics, 35 $37-43$. doi:10.1016/S0020-7462(98)00085-7

[33] Ganji, D.D., Amini, M. and Kolahdooz, A. (2008) Analytical investigation of hyperbolic Equations via He's methods. American. Journal of Engineering and Applied Science, 1, 399-407. 


\section{APPENDIX A}

Solution of the Eqs.7 and 8 using Homotopy perturbation method. In this Appendix, we indicate how Eqs.12 and 13 in this paper are derived. Furthermore, a Homotopy was constructed to determine the solution of Eqs.7 and 8.

$$
\begin{aligned}
& (1-p)\left[\frac{d^{2} U}{d X^{2}}\right]+p\left[\frac{d^{2} U}{d X^{2}}+\alpha_{1} U \frac{d^{2} U}{d X^{2}}-\gamma_{E} U\right]=0 \\
& (1-p)\left[\frac{d^{2} V}{d X^{2}}\right]+p\left[\frac{d^{2} U}{d X^{2}}+\alpha_{1} U \frac{d^{2} V}{d X^{2}}+\gamma_{E} U\right]=0
\end{aligned}
$$

and the initial approximations are as follows:

$$
\begin{gathered}
X=0, V=0, U=1 \\
X=1+\frac{\delta}{L}, V=0, U=1
\end{gathered}
$$

The approximate solutions of (A1) and (A2) are

$$
U=U_{0}+p U_{1}+p^{2} U_{2}+p^{3} U_{3}+\cdots \cdots
$$

and

$$
V=V_{0}+p V_{1}+p^{2} V_{2}+p^{3} V_{3}+\cdots \cdots
$$

Substituting Eqs.A5 and A6 into Eqs.A1 and A2 and comparing the coefficients of like powers of $p$

$$
\begin{aligned}
& p^{0}: \frac{d^{2} U_{0}}{d X^{2}}=0 \\
& p^{1}: \frac{d^{2} U_{1}}{d X^{2}}+\alpha_{1} U_{0} \frac{d^{2} U_{0}}{d X^{2}}-\gamma_{E} U_{0}=0 \\
& p^{2}: \frac{d^{2} U_{2}}{d X^{2}}+\alpha_{1} U_{0} \frac{d^{2} U_{1}}{d X^{2}}+\alpha_{1} U_{1} \frac{d^{2} U_{0}}{d X^{2}}-\gamma_{E} U_{1}=0
\end{aligned}
$$

and

$$
\begin{aligned}
& p^{0}: \frac{d^{2} V_{0}}{d X^{2}}=0 \\
& p^{1}: \frac{d^{2} V_{1}}{d X^{2}}+\alpha_{1} U_{0} \frac{d^{2} V_{0}}{d X^{2}}+\gamma_{E} U_{0}=0
\end{aligned}
$$

$$
p^{2}: \frac{d^{2} V_{2}}{d X^{2}}+\alpha_{1} U_{0} \frac{d^{2} V_{1}}{d X^{2}}+\alpha_{1} U_{1} \frac{d^{2} V_{0}}{d X^{2}}+\gamma_{E} V_{1}=0
$$

Solving the Eqs.A7 to A12, and using the boundary conditions (A3) and (A4), we can find the following results

$$
\begin{aligned}
U_{0}(X) & =1 \\
U_{1}(X)= & \frac{\gamma_{E}}{2}\left[X^{2}-\left(1+\frac{\delta}{L}\right) X\right] \\
U_{2}(X)= & \frac{1}{24}\left[\gamma_{E}^{2}\left(1+\frac{\delta}{\mathrm{L}}\right)^{2}+12 \alpha_{1} \gamma_{E}\left(1+\frac{\delta}{L}\right)\right] X+ \\
& \frac{1}{24}\left[\gamma_{E}^{2} X^{4}-2 \gamma_{E}^{2}\left(1+\frac{\delta}{L}\right) X^{3}-12 \alpha_{1} \gamma_{E} X^{2}\right]
\end{aligned}
$$

and

$$
V_{0}(X)=0
$$

$$
\begin{aligned}
V_{1}(X)= & \frac{\gamma_{E}}{2}\left[\left(1+\frac{\delta}{L}\right) X-X^{2}\right] \\
V_{2}(X)= & \frac{-1}{24}\left[\gamma_{E}^{2}\left(1+\frac{\delta}{\mathrm{L}}\right)^{3}+12 \alpha_{1} \gamma_{E}\left(1+\frac{\delta}{L}\right)\right] X Z+ \\
& \frac{1}{24}\left[12 \alpha_{1} \gamma_{E} X^{2}-\gamma_{E}^{2} X^{4}+2 \gamma_{E}^{2}\left(1+\frac{\delta}{L}\right) X^{3}\right]
\end{aligned}
$$

According to the HPM, we can conclude that

$$
\begin{aligned}
& U(X)=\lim _{p \rightarrow 1} U(X)=U_{0}+U_{1}+U_{2} \\
& V(X)=\lim _{p \rightarrow 1} V(X)=V_{0}+V_{1}+V_{2}
\end{aligned}
$$

Using Eqs.A13, A14 and A15 in Eq.A19 and Eqs.A16, A17 and A18 in Eq.A20, we obtain the final results as described in Eqs.12 and 13. 


\section{APPENDIX B}

Scilab program to find the solutions of the Eqs.7 and $\mathbf{8}$ function pdex 4

$\mathrm{m}=0$;

$\mathrm{x}=$ linspace $(0,1)$;

$\mathrm{t}=$ linspace $(0,100000)$;

sol=pdepe(m,@pdex4pde,@pdex4ic,@pdex4bc,x,t);

$\mathrm{u} 1=\operatorname{sol}(:,:, 1)$;

$\mathrm{u} 2=\operatorname{sol}(:,:, 2)$;

figure

$\operatorname{plot}(\mathrm{x}, \mathrm{u} 1(\mathrm{end}, \mathrm{:}))$

title('u1 $(\mathrm{x}, \mathrm{t})$ ')

xlabel('Distance $\mathrm{x}$ ')

ylabel('u1 (x,2)')

$\%$

figure

$\operatorname{plot}(\mathrm{x}, \mathrm{u} 2(\mathrm{end}, \mathrm{:}))$

title('u2(x,t)')

xlabel('Distance $\mathrm{x}$ ')

ylabel('u2(x,2)')

$\%$

function $[\mathrm{c}, \mathrm{f}, \mathrm{s}]=\operatorname{pdex} 4 \mathrm{pde}(\mathrm{x}, \mathrm{t}, \mathrm{u}, \mathrm{DuDx})$

$\mathrm{c}=[1 ; 1]$;

$\mathrm{f}=[1 ; 1] *$ DuDx;

$\mathrm{a}=0.1$;

$\mathrm{r}=1$;

$\mathrm{F}=(\mathrm{r} * \mathrm{u}(1)) /(1+\mathrm{a} * \mathrm{u}(1))$;

$\mathrm{S}=[-\mathrm{F} ; \mathrm{F}]$;

$\%$

function $\mathrm{u} 0=\operatorname{pdex} 4 \mathrm{ic}(\mathrm{x})$;

$\%$ create a initial conditions

$\mathrm{u} 0=[0 ; 1]$;

$\%$

function $[\mathrm{pl}, \mathrm{ql}, \mathrm{pr}, \mathrm{qr}]=\mathrm{pdex} 4 \mathrm{bc}(\mathrm{xl}, \mathrm{ul}, \mathrm{xr}, \mathrm{ur}, \mathrm{t})$

$\%$ create a boundary conditions

$\mathrm{pl}=[\mathrm{ul}(1)-1 ; \mathrm{ul}(2)]$

$\mathrm{ql}=[0 ; 0]$;

$\operatorname{pr}=[\operatorname{ur}(1)-1 ; \operatorname{ur}(2)] ;$

$\mathrm{qr}=[0 ; 0]$ 